\title{
RESIDUOS SÓLIDOS DOMICILIARIOS: CARACTERIZACIÓN Y ESTIMACIÓN ENERGÉTICA PARA LA CIUDAD DE CHIMBOTE
}

\author{
Serapio A. Quillos Ruiz ${ }^{a^{*}}$, Nelver J. Escalante Espinozaa ${ }^{a}$ Daniel A. Sánchez Vaca ${ }^{a}$, \\ Luis G. Quevedo Novoa ${ }^{b}$, Ronal A. De La Cruz Araujo ${ }^{a}$
}

\begin{abstract}
RESUMEN
El manejo y la disposición final de los Residuos Sólidos Domiciliarios (RSD) presentan desafíos y, a la vez, oportunidades para los países en vías de desarrollo y con poco progreso científico-tecnológico en este ámbito. En este trabajo se evaluó la caracterización de los RSD para realizar la estimación del potencial energético contenido en los Residuos Sólidos Orgánicos Domiciliarios (RSOD) de la ciudad de Chimbote. La muestra de estudio consistió en 60 viviendas, distribuidas en tres niveles socioeconómicos, tal como establece la metodología estadística del Ministerio del Ambiente del Perú. Así, entonces, se obtuvo una generación per cápita de RSD de 0,425 kg/hab./día, donde los RSOD representan el 69,03\% $(0,297 \mathrm{~kg} / \mathrm{hab}$./día) de los RSD. Basado en estos resultados, el total de los RSOD alcanza el 69,8 Tn/día. Finalmente, se realizó una evaluación para estimar la recuperación energética contenida en los RSOD a través de la incineración, obteniéndose una generación de 15,33 $M W$ al año 2017, situación que representaría un aumento del $8 \%$ durante la década 2017 27. Al recuperar la energía contenida en los RSOD se puede contribuir a la reducción del consumo de combustibles fósiles, así como también a la disminución del impacto ambiental, evitando principalmente las emisiones de metano.
\end{abstract}

Palabras clave: residuo sólido domiciliario, energía renovable, metano, biogás

\section{SOLID DOMICILIARY RESIDUES: CHARACTERIZATION AND ENERGY ESTIMATION FOR THE CITY OF CHIMBOTE}

\begin{abstract}
The management and final disposal of Residential Solid Waste (RSD) present challenges and, at the same time, opportunities for the developing countries, and with little scientifictechnological progress in this area. In this work, the characterization of the RSD was evaluated to estimate the energy potential contained in the Residential Solid Organic Residues (RSOD) of the city of Chimbote. The study sample consisted of 60 dwellings, distributed into three

a* Escuela Profesional de Ingeniería Mecánica. Universidad Nacional del Santa. Nuevo Chimbote. Ancash, Perú. Código postal 02710.squillos@uns.edu.pe

b Escuela Profesional de Ingeniería Mecánica. Universidad Nacional de Trujillo. Trujillo. Perú. Código postal 13007.
\end{abstract}


socioeconomic levels, as established by the statistical methodology of the Ministry of the Environment of Peru. Thus, a per capita generation of RSD of $0.425 \mathrm{~kg} / \mathrm{person} /$ day was obtained, where the RSOD represent $69.03 \%$ (0.297 kg/person/day) of the RSD. Based on these results, the total of the RSOD reaches 69.8 tons/day. Finally, an evaluation was carried out to estimate the energy recovery contained in the RSOD through incineration, obtaining a generation of $15.33 \mathrm{MW}$ by 2017 , a situation that would represent an increase of $8 \%$ during the decade 2017-27. Recovering the energy contained in the RSOD can contribute to the reduction of the fossil fuel consumption, as well as the reduction of the environmental impact therein, mainly avoiding methane emissions.

Key words: Residential solid waste, renewable energy, methane, biogas.

\section{INTRODUCCIÓN}

En la actualidad, la fuente principal para la obtención de energía es a partir de los combustibles convencionales como el petróleo, carbón y gas natural. En consecuencia, sus reservas son altamente explotadas, generando una elevación en sus precios, un posible agotamiento, vulnerabilidad del suministro y un incremento del calentamiento global causado por las emisiones de $\mathrm{CO}_{2}$ debido al alto consumo de los mismos ${ }^{1}$. Además, el continuo crecimiento de la población mundial incrementa la demanda energética a niveles no proyectados en el pasado ${ }^{2}$. Esto obliga a buscar nuevas fuentes de generación de energía, las cuales se encontrarían en el desarrollo de las energías renovables ${ }^{2,3}$. La tecnología necesaria para el aprovechamiento de estas últimas se encuentra en pleno desarrollo, pero aún presenta problemas financieros para su implementación ${ }^{3}$. Los estudios indican que estas formas de generación cada vez se vuelven más eficientes, económicas y autosostenibles para las diversas necesidades (confort, transporte, cocina, industria y electricidad) ${ }^{1}$.

El Programa Especial para el Aprovechamiento de Energías Renovables (PEAER) ${ }^{4}$ de México, señala las principales áreas para un sistema energético sostenible y establece que el mayor potencial teórico se encuentra en la energía solar, eólica, recursos geotérmicos y la biomasa. Esta última está compuesta por bagazo de caña, licor negro y biogás, el cual se encuentra conformado por lodos residuales, residuos agropecuarios, residuos industriales y residuos sólidos municipales (RSM). Los RSM son la mayor fuente de producción de biogás y la segunda fuente de generación eléctrica de la biomasa ${ }^{4}$. Por lo tanto, los RSM son una fuente potencial de energía renovable.

En los países sudamericanos los procesos de recolección, tratamiento, aprovechamiento y disposición final de los RSM son realizados con tecnologías inadecuadas, pues la participación de los sectores informales es muy frecuente debido a que representa su principal sustento económico ${ }^{3}$.

La ciudad de Chimbote representa uno de los puntos más críticos con RSM en el Perú, debido a que su disposición final es un botadero a cielo abierto, albergando animales carroñeros 
que son una fuente de transmisión de enfermedades para la población cercana. El botadero municipal se ubica entre los límites urbanos de Chimbote y Coishco, con un área de 16 hectáreas, donde son depositados 1 060,23 Tn semanalmente. La generación diaria per cápita (en adelante referido sólo como generación per cápita o GPC) de RSM fue de 0,64 kg/hab./ día al año 2015, que es cercana al promedio nacional ${ }^{5}$.

Los RSM son un grave problema de contaminación que afecta el medio ambiente y la vida humana. Por esta razón, para la optimización de la recuperación energética desde los residuos se debe tener una adecuada caracterización para cada una de las fuentes que contribuyen a estos RSM, tales como residuos sólidos domiciliarios (RSD), residuos sólidos comerciales, residuos sólidos agrícolas, etc ${ }^{6}$. Adicionalmente, otro factor es el estudio de la relación entre el nivel de vida (clasificación socioeconómica) y el promedio de residuos generados por cada persona ${ }^{7}$.

Los esfuerzos por resolver el problema actual son pocos. Sólo se conoce una institución que desea invertir en los RSM con el fin de obtener productos a través del reciclaje. Del mismo modo, se tendría el efecto contaminante porque lo que se puede reciclar típicamente es menos del $50 \%{ }^{3}$. Para aprovechar de alguna forma la fracción de RSD que no se puede reciclar (principalmente componente orgánico), se deben evaluar dos aspectos básicos: la cantidad que se genera y su contenido energético ${ }^{6}$. El grado de aprovechamiento de este contenido energético depende del proceso de conversión de la energía química contenida en la basura a energía útil; esos procesos son, por ejemplo, los de tipo biológico y los de tipo térmico ${ }^{6}$.

La caracterización de la composición de los RSD nos permite determinar la cantidad de residuos sólidos orgánicos domiciliarios (RSOD) que serán convertidos a biogás por fermentación anaeróbica o por incineración para generar energía útil y así poder determinar su potencial energético ${ }^{6}$. Al conocer la caracterización de los RSD se puede establecer el mercado de reciclaje y el potencial energético ${ }^{5}$. Además, es necesario aclarar que las fuentes de RSD forman la mayor parte de RSM y son más homogéneas en generación per cápita comparadas con otras fuentes que son fluctuantes según el rubro (por ejemplo, hoteles, industrias o mercados $)^{5}$. Es importante mencionar además que la disposición de los RSM se presenta como un problema político y tecnológico, debido a falta de decisión para establecer el relleno sanitario en el lugar apropiado, donde se debe complementar con un marco legal proporcionado por las entidades involucradas (MINAM, Sigersol, municipalidades) ${ }^{3}$. También se requiere estudios consistentes de los RSM para tener claro los conceptos de generación per cápita y caracterización de residuos. De esta manera, poder desarrollar proyectos destinados a corregir las ineficiencias en el manejo y disposición final de los RSM, con el objetivo de que a mediano y a largo plazo se pueda establecer programas educativos ambientales ${ }^{3}$.

SIGERSOL reporta, a través de su portal web, la caracterización de los RSM de la ciudad de Chimbote, de los años 2011 al 2015, en base a la ley № 1278 Ley de Gestión Integral de Residuos Sólidos y su Reglamento $N^{\circ}$ 174-2017-MINAM, pero no expone de manera explícita la metodología usada. Esto indica que es necesario tener la información actualizada $\mathrm{y}$, sobre todo, particularizada para los $\mathrm{RSD}^{5}$. 
En la presente investigación se ha realizado la caracterización de los RSD de la ciudad de Chimbote, para luego realizar una estimación del potencial energético contenido en los RSOD. Para esto se ha determinado la población actual, con la cual se ha obtenido el tamaño de la muestra (cantidad de viviendas). Se ha calculado la generación per cápita diaria global (evaluada por una semana); así como el porcentaje de cada uno de los componentes de los RSD. Con la información previa y las propiedades físico-químicas de los RSOD, hemos realizado una estimación energética para la década 2017-27.

Este artículo está organizado como sigue. En la sección de materiales y métodos se describe la metodología seguida para caracterizar los RSD y realizar la estimación energética de los RSOD. En la sección de resultados mostramos la caracterización realizada en una semana y los valores promedio de GPC, así como valoración porcentual de energía a obtener. Resumimos nuestros principales hallazgos en la sección de conclusiones.

\section{MATERIALES Y MÉTODOS}

\section{Zona de estudio}

La ciudad de Chimbote está situada en la costa norte del Perú $\left(9^{\circ} 05^{\prime} 07^{\prime \prime} \mathrm{S}, 78^{\circ} 34^{\prime} 41^{\prime \prime}\right.$ O) y tiene una superficie de $1467 \mathrm{~km}^{2}$ (figura 1). En el año 2007 tenía una población de 215817 habitantes, con una tasa de crecimiento de $0,8 \%$ anual, reportado por el Instituto Nacional de Estadística e Informática (INEI) del Perú; la población urbana comprende el 96,13\% y la rural el 3,87\%, contando con un total de 49903 viviendas al año $2007^{8}$. Es la ciudad de mayor superficie de la provincia del Santa $(46,2 \%)$; ha tenido un rápido crecimiento demográfico y económico dado por el desarrollo de la pesca, la cual contribuye con el 18,1 del PBI sectorial (3,3 PBI nacional) y es una de las 10 ciudades más importantes del país. Pero, a la vez, con alta contaminación, por no tener implementada una gestión aceptable para los RSM que genera ${ }^{8}$.

\section{Tamaño de muestra}

La proyección de la población para un año requerido se determina en función de la ecuación ${ }^{9}$

$$
P_{F}=P_{i}(1+\mathrm{r})^{t}
$$

donde $P_{F}$ es la población final proyectada, $P_{i}$ representa la población inicial del último censo nacional, $r$ es la tasa de crecimiento anual inter censal y t es el número de años que se desea proyectar a la población, a partir de la población inicial. 


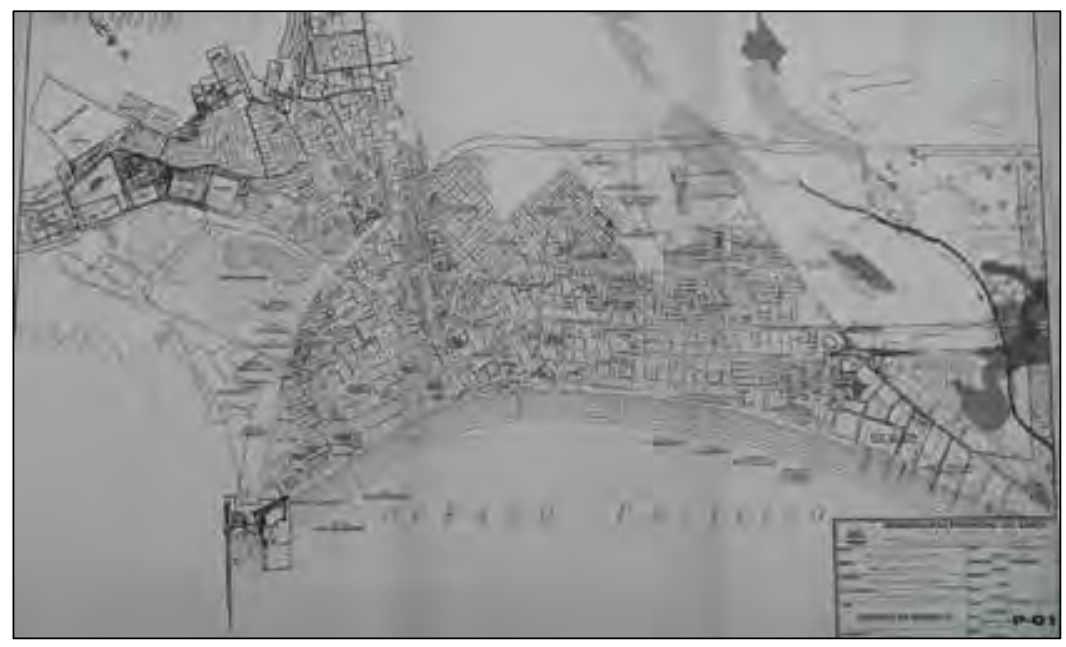

Figura 1. Mapa de la ciudad de Chimbote (Fuente: mapa tomado con autorización de la Municipalidad Provincial del Santa)

Para la caracterización de los RSD se usa una metodología en función del procedimiento estadístico, donde la muestra representativa de viviendas es obtenida por la siguiente ecuación ${ }^{9}$ :

$$
n=\frac{\mathrm{Z}_{1-\alpha / 2}^{2} N \sigma^{2}}{(N-1) \mathrm{E}^{2}+\mathrm{Z}_{1-\alpha / 2}^{2} \sigma^{2}}
$$

siendo $n$ es el tamaño de la muestra, $N$ el número de viviendas de la ciudad (49 903) ${ }^{8}, \sigma$ la desviación estándar $(0,250 \mathrm{~kg} / \mathrm{hab}$./dia $)$ esperada ${ }^{9}$, es decir, en cuánto se espera que varíe la GPC de los RSD. Z1- $\alpha / 2$ representa el nivel de confianza $(1,96)$, se considera este valor para obtener un confianza del $95 \%$ en los valores de generación de RSD. $E$ es el error permisible del $10 \%$ la estimación de la GPC nacional de RSM $(0,70 \mathrm{~kg} / \mathrm{hab} . / \mathrm{dia})^{10}$, resultando $E=$ $0,07 \mathrm{~kg} / \mathrm{hab}$. $/ \mathrm{di}^{\mathrm{a}}{ }^{9}$. La toma de muestras se realiza cumpliendo con los procedimientos y lineamientos internacionales aceptados por la legislación ambiental peruana ${ }^{9}$. La aplicación del método de análisis de residuos sólidos y la metodología para el desarrollo del estudio, determina la eficiencia de separación y permite la recuperación de los RSD generados ${ }^{9}$, que comprenden: papel y cartón, plásticos, madera, textil, orgánicos, metales, vidrio y otros. $\mathrm{Al}$ año 2017 Chimbote tuvo una población de 238396 habitantes obtenida mediante la ecuación (1) y el tamaño de la muestra (cantidad de viviendas) se calculó usando la ecuación (2), adicionando un $15 \%$ para las muestras no consistentes ${ }^{9}$. La cantidad de muestras resultantes fue 60 y se repartió en tres estratos socioeconómicos alto $(A)$, medio $\left(B\right.$ y $C$ ) y bajo $(D)^{8,9}$ representando cada estrato, por su porcentaje de contribución al total9. Esto en cumplimiento a lo establecido por la norma nacional y que es de aplicación frecuente en la literatura revisada 9 . Como es recomendado en las normas, la tarea de caracterización es realizada durante ocho días consecutivos en las viviendas seleccionadas aleatoriamente en cada estrato, descartando la data del primer día y siendo útil solo los datos de siete días ${ }^{9}$. En la tabla 1 se evidencia la 
distribución de muestras por niveles socioeconómicos ${ }^{9,10}$. En esta se observa que la mayoría de viviendas fueron seleccionadas en el estrato socioeconómico bajo (D) debido a que es el estrato mayoritario de la ciudad $(43,5 \%)$.

Tabla 1. Distribución de las muestras de RSD en los tres niveles socioeconómicos.

\begin{tabular}{cccc}
\hline \multicolumn{2}{c}{ Estrato socioeconómico } & Población (\%) & $\mathbf{N}^{\mathbf{0}}$ de muestras \\
\hline Alto & $A$ & 24,1 & 14 \\
Medio & $B C$ & 32,4 & 19 \\
Bajo & $D$ & 43,5 & 27 \\
\hline
\end{tabular}

Para calcular la GPC de cada domicilio y por estratos socioeconómicos utilizamos las ecuaciones:

$$
\begin{gathered}
G P C_{i}=1 / 7 N_{h} \sum_{p=1}^{p=7} m R S D_{p} \\
G P C_{e}=1 / N_{v} \sum_{i=1}^{i=N_{v}} G P C_{i}
\end{gathered}
$$

donde GPCi representa GPC en $\mathrm{kg} / \mathrm{hab}$./día de RSD por vivienda sin importar el estrato socioeconómico, $m R S D_{p}$ representa la masa de los residuos de la vivienda (en $\mathrm{kg}$ ) para el día $p, N_{h}$ representa el número de habitantes de la vivienda; $G P C_{e}$ es el resultado del per cápita del estrato socioeconómico, para el número de viviendas (muestras), $N_{v}$, correspondientes al estrato $e(e=A, B C, D)$ según la tabla 1 .

Finalmente, para evaluar la generación per cápita de la ciudad de Chimbote, $G P C d$, usamos la ecuación:

$$
G P C_{d}=\sum_{e=1}^{e=3} G P C_{e} P o_{e}
$$

donde la $G P C_{d}$ se mide en $\mathrm{kg} / \mathrm{hab}$./día y $\mathrm{Po}_{e}$ es el porcentaje poblacional del estrato. La ecuación (5) determina la GPC de los RSD de Chimbote. De la misma forma las ecuaciones (3), (4) y (5) determinan los per cápita de los RSOD, utilizando solamente los componentes orgánicos en lugar del total.

La labor de campo comprendió la recolección de los residuos de las viviendas seleccionadas en forma aleatoria para cada estrato socioeconómico, luego los residuos son llevados al laboratorio para la caracterización y pesado de cada componente en la balanza electrónica marca Aro de $5 \mathrm{~kg}$ de capacidad. Realizándose seguidamente la evaluación de las características físico-químicas de los residuos tal como se describe a continuación.

\section{Análisis físico-químico}

En el Laboratorio de Fisicoquímica de la Universidad Nacional del Santa (UNS), se determinó el poder calorífico superior $(P C S)$ de los RSOD, donde se pesó $1 \mathrm{~g}$ de muestra seca (pulverizada 
y homogénea), llevándose en forma de pastilla al crisol de la bomba calorimétrica marca $P A R R$, modelo 3115, adicionando el oxígeno a 18 atm para la combustión de la muestra. Para calcular el poder calorífico inferior $(P C I)$ usamos la relación ${ }^{11}$.

$$
P C I=P C S-\frac{w}{100} 600
$$

Donde ambos el $P C S$ y el $P C I$ están en $\mathrm{kJ} / \mathrm{kg}$ y $w$ es la humedad en (\%). Se tomó 10 muestras independientes provenientes de los diferentes estratos y los valores promedio obtenidos se reportan en la sección de resultados.

\section{Potencial energético}

Los RSOD son una fuente de energía, la cual se puede obtener por incineración o digestión anaeróbica, principalmente. En la figura 2 representamos los dos procesos principales de transformación de los RSOD a energía útil.

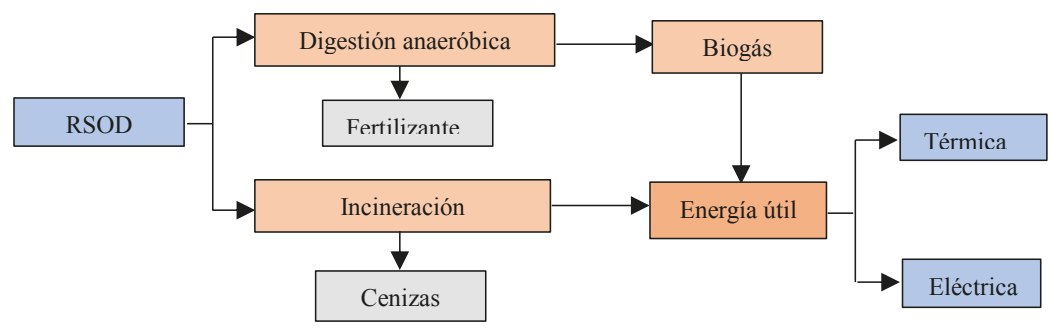

Figura 2. Procesos para la transformación de los RSOD a energía útil

La producción de energía secundaria de los RSOD se realiza por medio de procesos tecnológicos que permiten transformarla a energía útil (térmica o eléctrica), lo cual se viene aplicando en ciudades medias y grandes metrópolis como sucede en Europa, Asia y Estados Unidos. Esto se presenta como una opción de aprovechamiento de energía renovable ${ }^{12}$. Entonces, es necesario determinar el potencial energético en $M W$ para tener una estimación del recurso renovable en los próximos años de los RSD y RSOD utilizando las mejores tecnologías disponibles ${ }^{12}$. Para este fin utilizamos las relaciones:

$$
\begin{aligned}
\mathrm{GD} & =\left(G P C_{\text {org }}\right)\left(P_{F}\right) \\
\mathrm{Pe} & =(\mathrm{PCI})(\mathrm{GD})
\end{aligned}
$$

donde $G D$ es la generación diaria de RSOD en $\mathrm{kg} / \mathrm{dia}$ de la ciudad, $G P C_{\text {org }}$ es la generación per cápita de los residuos orgánicos domiciliarios en $\mathrm{kg} / \mathrm{hab}$./día. El $\mathrm{Pe}$ es el potencial energético en $\mathrm{kJ} / d i a$. Para la generación de energía útil por incineración usando el método de cogeneración se puede producir energía eléctrica con eficiencia de aprovechamiento que está entre $20-30 \%$ y energía térmica de alrededor de $50 \%$ con pérdidas del proceso que varían entre $20-25 \%{ }^{13}$. 


\section{RESULTADOS Y DISCUSIÓN}

\section{Caracterización de los RSD}

La caracterización diaria de RSD de la ciudad de Chimbote se muestra en la figura 3. En esta, la generación diaria per cápita de $\mathrm{RSD}, G P C_{d}$, y la generación diaria per cápita de RSOD, $G P C_{\text {org }}$, durante los siete días de estudio se muestran en las figuras $3 a$ y $3 b$, respectivamente. En la figura se observa los cambios diarios del promedio de GPC al igual que los cambios en sus desviaciones estándar, pero estas fluctuaciones no tienen tendencia notable a crecer o a disminuir. Esto implica que las muestras diarias son estadísticamente independientes entre sí, justificando la obtención de un promedio semanal. La desviación estándar máxima obtenida para GPCd fue de $0,241 \mathrm{~kg} / \mathrm{hab}$./día (ver día 6 en figura $3 a$ ) y para $G P C_{\text {org }}$ fue de $0,203 \mathrm{~kg} /$ hab./día (ver día 5 en figura $3 b$ ). Estas desviaciones obtenidas son menores a la asumida $(0,250 \mathrm{~kg} / \mathrm{hab} . / \mathrm{di} a)$ para calcular el tamaño de la muestra, indicando que no es necesario recalcular el tamaño de esta para actualizar la caracterización. Estas desviaciones estándar están en el rango observado, por ejemplo, en el distrito de Ate en Lima ( 0,318 $\mathrm{kg} / \mathrm{hab}$./ dia $)^{14}$.

Es importante notar que la $G P C_{\text {org }}$ representa más del $50 \%$ de la $G P C d$ (figura 3), indicando el alto contenido de componente orgánico que se puede aprovechar para generar energía útil. Porcentajes más detallados de cada uno de los componentes de los RSD son mostrados más adelante como valores promedio de los siete días de caracterización.
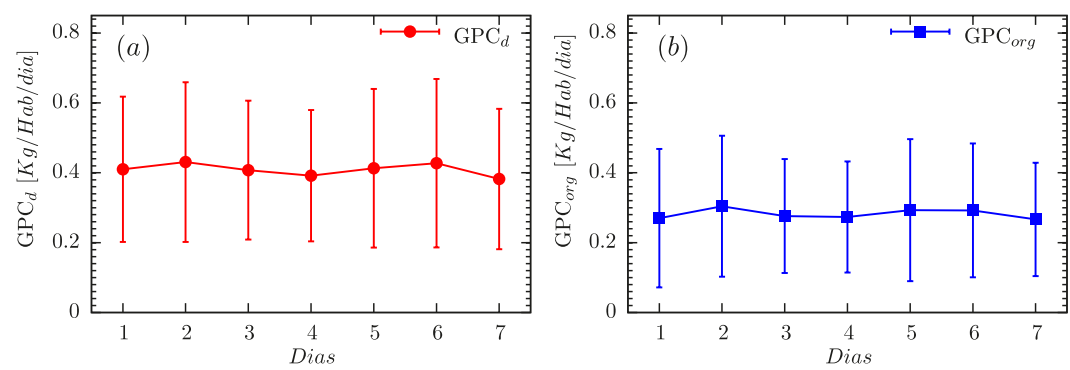

Figura 3. Caracterización diaria de RSD para la ciudad de Chimbote para el año 2017. (a) Generación per cápita total de RSD, $G P C_{d}$, y (b) generación per cápita de RSOD, $G P C_{\text {org }}$, contenidos en los RSD.

La tabla 2 muestra la producción per cápita ponderada, $G P C_{d}$, con ponderación dada por la contribución porcentual de cada estrato socioeconómico, $P_{o}$, para la ciudad de Chimbote. Estos resultados son valores promedio de los siete días de caracterización. El estrato $A$ con nivel socioeconómico alto muestra una mayor generación per cápita $(0,532 \mathrm{~kg} / \mathrm{hab}$./día $)$ respecto a los otros, pero como su contribución porcentual es menor respecto a los estratos medio $B C$ y bajo $D$, el promedio per cápita global $\left(G P C_{d}=0,425 \mathrm{~kg} / \mathrm{hab} . /\right.$ día $)$ es predominado por el estrato medio $(\mathrm{GPCBC}=0,429 \mathrm{~kg} / \mathrm{hab} . /$ día $)$. Esto indica que el aumento de la $G P C_{d}$ depende principalmente del nivel socioeconómico medio, mientras que la distribución porcentual de estratos no cambie. Los resultados son cercanos a los reportados para el distrito 
de Ate, Lima, con per cápita por estratos de 0,530 0,710 y 0,665 $\mathrm{kg} / \mathrm{hab}$. $/$ día en los niveles $\mathrm{A}$, $B$ y $C$, respectivamente ${ }^{14}$. Estos resultados son ligeramente mayores a los que hemos obtenido y se justifican por tener un mejor nivel socioeconómico. Esto confirma que los residuos aumentan con la calidad de vida ${ }^{7}$.

Tabla 1. Generación per cápita ponderada de residuos sólidos domiciliarios de Chimbote.

\begin{tabular}{clccc}
\hline $\begin{array}{c}\text { Estrato } \\
\text { Socioeconómico }\end{array}$ & $\begin{array}{c}\boldsymbol{G P C _ { \boldsymbol { e } }} \\
(\boldsymbol{k g} / \boldsymbol{h a b} \text { /día })\end{array}$ & $\begin{array}{c}\boldsymbol{P o}_{\boldsymbol{e}} \\
\mathbf{( \% )}\end{array}$ & $\begin{array}{c}\boldsymbol{G P C}_{\boldsymbol{d}} \\
(\boldsymbol{k g} / \boldsymbol{h a b} \text { /día })\end{array}$ \\
\hline Alto & $A$ & 0,532 & 24,10 & \\
Medio & $B C$ & 0,429 & 32,40 & 0,425 \\
Bajo & $D$ & 0,363 & 43,50 & \\
\hline
\end{tabular}

El GPCd mostrado en la tabla 2 está por encima de los reportados para los distritos de Pachacámac $(0,37 \mathrm{~kg} / \mathrm{hab} . /$ día $)$, Punta Negra $(0,26 \mathrm{~kg} / \mathrm{hab}$./día); pero cercanos a los de Lurigancho (0,44 kg/hab./dia), Puente Piedra (0,49 kg/hab./dia) y Santa Rosa (0,47 kg/hab./ día); pero mucho menores que en los distritos de San Miguel (1,23 kg/hab./dia), Santiago de Surco $(1,15 \mathrm{~kg} / \mathrm{hab} . /$ dia $)$ y otros distritos de la ciudad de Lima de mayor poder adquisitivo, como reporta el Sistema Nacional de Información Ambiental (SINIA) del Perú. Esta comparación refuerza lo enunciado anteriormente.

El promedio per cápita nacional es de $0,7 \mathrm{~kg} / \mathrm{hab}$. $/ \mathrm{di}^{10}{ }^{10}$. Chimbote se encuentra en un valor menor al nacional pero superior a los valores reportados para Amazonas $(0,3 \mathrm{~kg} / \mathrm{hab}$./día) y Huancavelica $(0,2 \mathrm{~kg} / \mathrm{hab} . / \mathrm{di} a)^{10}$. Indicando todavía un alto grado de generación de RSD.

La tabla 3 muestra por separado la generación per cápita del componente en mayor porcentaje contenido en los RSD, nos referimos al componente orgánico (RSOD). Esta muestra que los RSOD representan un $69 \%\left(100 \% G P C_{\text {org }} / G P C d\right)$ de los RSD. Una distribución detallada de los componentes del RSD se reporta en la tabla 4.

Tabla 3. Generación per cápita de residuos sólidos orgánicos domiciliarios de Chimbote.

\begin{tabular}{|c|c|c|c|}
\hline $\begin{array}{c}\text { Estrato } \\
\text { Socio económico }\end{array}$ & $\begin{array}{c}G P C_{e, \text { org }} \\
(\mathrm{kg} / \mathrm{hab} . / \text { día })\end{array}$ & $\begin{array}{r}P o_{e} \\
(\%)\end{array}$ & $\begin{array}{c}\text { GPC } C_{\text {org }} \\
(\mathrm{kg} / \mathrm{hab} . / \text { día })\end{array}$ \\
\hline Alto $A$ & 0,374 & 24,10 & \\
\hline Medio $B C$ & 0,315 & 32,40 & 0,293 \\
\hline Bajo $\quad D$ & 0,233 & 43,50 & \\
\hline
\end{tabular}

Tabla 4. Caracterización de los RSD

\begin{tabular}{clr}
\hline $\mathbf{N}^{\mathbf{0}}$ & Subproducto & $\mathbf{\%}$ \\
\hline 1 & Orgánico & 69,03 \\
2 & Papel y cartón & 12,70 \\
3 & Plásticos & 10,58 \\
4 & Madera & 0,23 \\
5 & Textil & 0,47 \\
6 & Metales & 1,18 \\
7 & Vidrio & 2,82 \\
8 & Otros & 2,99 \\
\hline & & 100,00
\end{tabular}


En la tabla 4 se observa que los RSOD representan el $69 \%$ del total de RSD. El material potencialmente reciclable (papel y cartón, plásticos, madera, textil, metales y vidrio) representa el $28 \%$ y el $3 \%$ es material no reciclable y no orgánico (denominado como “otros" en la tabla 4). A nivel nacional, estos porcentajes son: $55 \%$ material orgánico, $20 \%$ materiales reciclables y $25 \%$ materiales no reciclables ${ }^{15}$; pero incluye residuos sólidos de otras fuentes que en total forman los RSM. El elevado porcentaje de componente orgánico lo atribuimos a que solo estamos caracterizando los residuos sólidos domiciliarios, donde el material orgánico es el principal componente (restos de alimentos, frutas y plantas). Este valor alto de componente orgánico es similar a lo reportado para el distrito de Ate en Lima $(60,58 \%)^{14}$ y Limoncocha en Ecuador $(73 \%)^{11}$. Además, es comparable con altos porcentajes reportados para otros países en los cuales el componente orgánico puede superar el $70 \%$ de los $\mathrm{RSM}^{2,3}$. Por consiguiente, es importante conocer el porcentaje del RSOD, para lo cual se requiere realizar la caracterización con bastante precisión, de esta manera determinar la estimación del aprovechamiento de la energía. La ciudad de Chimbote cuenta con una fracción orgánica de $\sim 70 \%$, que es altamente representativa y atractiva para proyectos de inversión con objetivos de recuperación energética y al cuidado del medio ambiente.

\section{Estimación del aprovechamiento energético de los RSOD}

Se requiere aprovechar los RSOD aplicando la recuperación de energía contenida en los residuos, proceso conocido como basura a energía o Waste to Energy (WTE) ${ }^{2,12}$. Solamente usamos el componente orgánico porque los otros elementos energéticos son aprovechados para el reciclaje, cuya actividad es sustento para las familias de bajo recursos y además regulada por la ley peruana No 29419. En la tabla 5 tenemos las propiedades físico-químicas promedio de los RSOD para 10 muestras independientes.

Tabla 5. Poder calorífico superior $(P C S)$ y poder calorífico inferior $(P C I)$ de los RSOD. Se muestra valores promedio evaluados para 10 muestras independientes.

\begin{tabular}{cccc}
\hline $\boldsymbol{P C S}(\boldsymbol{k J} / \mathbf{k g})$ & $\boldsymbol{P C I}(\mathrm{kJ} / \mathbf{k g})$ & Humedad $(\%)$ & Densidad $(\boldsymbol{g} / \mathbf{m} \boldsymbol{l})$ \\
\hline 19414 & 18956 & 76,258 & 1,031 \\
\hline
\end{tabular}

Los valores de $P C S$ y $P C I$ de los RSOD son superiores al valor recomendado (13 $000 \mathrm{~kJ} / \mathrm{kg})$ para lograr una eficiencia energética de $\sim 30 \%$ en un incinerador de residuos para producir electricidad y de $\sim 50 \%$ para aprovechar como energía térmica ${ }^{11}$. La generación nacional de RSM es en promedio $22390 \mathrm{Tn} /$ día (Año 2015), que se recogen en las municipalidades del país ${ }^{10}$. Al año 2017 la cantidad de RSD obtenida para la ciudad de Chimbote resultó en $101,13 T n-R S D /$ día calculadas usando la $G P C_{d}$ mostrado en la tabla 2 y usando la ecuación 7. Este total diario de RSD de la ciudad de Chimbote corresponde al 0,5\% del promedio nacional. De la misma forma, aplicamos las ecuaciones 1,7 y 8 para obtener los resultados mostrados en la tabla 6. En esta tabla, se observa que al 2017 se puede obtener 15,33 $\mathrm{MW}$ de potencia de generación $(P G)$ usando los RSOD.

La WTE que se puede estimar para los próximos años (2017-2027), depende de los factores que afecten a la generación per cápita de RSOD. Estos factores son el aumento de la 
población, calidad de vida y gestión de los residuos, principalmente. Aclaramos que para los próximos años solamente podemos realizar una estimación, debido a que el periodo de evaluación no es suficiente para realizar una proyección.

Tabla 6. Potencial energético $(P E)$ y potencia de generación $(P G)$ de los RSOD estimada para el año 2017

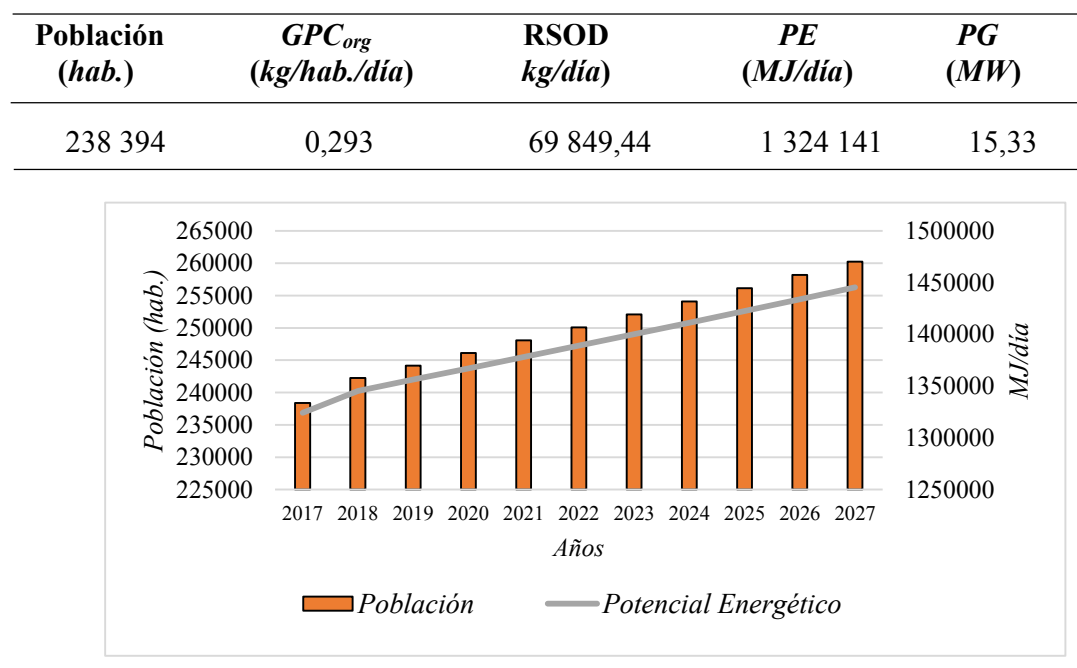

Figura 4. Estimación energética de los RSOD, desde el año 2017 hasta el año 2027, para la ciudad de Chimbote. Las barras verticales representan la cantidad de habitantes para cada año, mientras que la línea continua representa el potencial energético en MJ/día.

En la figura 4 se muestra la estimación del potencial energético evaluado con el incremento poblacional (según ecuación 1) y asumiendo un valor constante de generación per cápita $\left(G P C_{\text {org }}=0,293 \mathrm{~kg} / \mathrm{hab} . /\right.$ día $)$. Además, mostramos el crecimiento poblacional, el cual es el factor que causa el incremento del potencial energético. Se observa que a 10 años el potencial energético puede aumentar desde $1324141 \mathrm{MJ} /$ dia $(15,33 \mathrm{MW})$ hasta $1433968 \mathrm{MJ} /$ día $(16,60 \mathrm{MW})$, lo que representa un incremento de $8 \%$ respecto al potencial actual (al 2017). Obviamente, este valor puede ser modificado si cambia la generación per cápita de la ciudad. Pensando en aprovechar la energía obtenida por incineración y utilizando cogeneración, la figura 5 muestra la variación para la energía térmica y eléctrica estimada para 10 años. En esta se muestra que mediante la cogeneración se ofertaría una potencia de generación térmica de 7,66 $M W$ y eléctrica de 4,60 MW al 2017, ambas con un incremento leve pero continuo hasta alcanzar un $8 \%$ de incremento dentro de 10 años. La primera opción de WTE lo tiene la incineración, cuya tecnología está más desarrollada que otros métodos; además reduce grandemente el volumen inicial y en el futuro permitiría procesar los residuos reciclables de ser el caso ${ }^{2}$. Estimaciones similares se podrían realizar en caso de usar el método de digestión anaeróbica (figura 2) para generar biogás. El estudio de este método está fuera del alcance de este trabajo. El lector interesado podría revisar los modelos europeos y americanos para realizar una estimación energética razonable con este método ${ }^{2}$. 


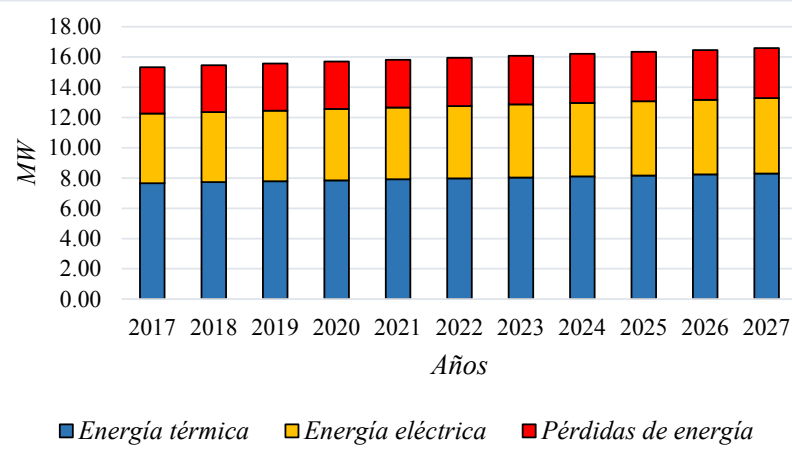

Figura 5. Estimación energética de generación de los RSOD para la ciudad de Chimbote usando cogeneración. Las barras azules representan el estimado de energía térmica que se produciría (50\% del $P E)$, las amarillas la energía eléctrica $(30 \%$ del $P E)$ mientras las rojas representan el estimado de las pérdidas que siempre estarían presentes $(20 \%$ del $P E)$.

El proceso de incineración se ha desarrollado bastante a diferencia de la digestión anaeróbica, la cual tiene como producto intermedio el biogás, para luego llegar a la combustión. Pero en ambos procesos persiste el problema de la polución, porque toda combustión genera emisiones de $\mathrm{CO}_{2}$, causantes del efecto invernadero, cuando la combustión es completa. En la combustión incompleta, produce además emisiones de $\mathrm{CO}$, que afectan al ser humano y, en caso extremo, causar la muerte. Una importante ventaja de ambos procesos es que evitan las emisiones de metano (gas de efecto invernadero) y permiten la recuperación energética, dando utilidad a los residuos domiciliarios considerados inútiles.

\section{CONCLUSIONES}

En el presente trabajo se ha caracterizado los residuos sólidos domiciliarios de la ciudad de Chimbote y se ha estimado la energía que se podría recuperar del componente orgánico contenido en estos. Nuestras muestras fueron separadas por componentes como típicamente se hace en otras ciudades y/o países, tales como materia orgánica, papel y cartón, metales, plásticos, madera, textiles, vidrio y otros. Se obtuvo una alta producción per cápita del componente orgánico $(69,03 \%$ en peso de los RSD) respecto al promedio nacional (55\% en peso de los RSM ${ }^{15}$, resultados cercanos a los obtenidos por otras ciudades.

Los plásticos, juntos con el papel y cartón, son los otros componentes que presentan un alto porcentaje $(\sim 12 \%)$, mientras que los demás componentes no superan el $10 \%$ de la generación per cápita global, que resultó en $0,425 \mathrm{~kg} / \mathrm{hab}$./día. Claramente, si evaluáramos otros RSM tales como provenientes de restaurantes, mercados, industrias metalmecánicas, hoteles, etc., obtendríamos diferentes composiciones. Esto muestra una necesidad apremiante de tener una caracterización específica de los RSD y actualizada para cada ciudad del Perú, de tal manera que se pueda realizar una estimación más razonable del potencial energético nacional proveniente de estos residuos. 
En nuestros países en vías de desarrollo la recuperación "energética" del papel, cartón, plásticos, metales y vidrio se realiza a través del reciclaje, el cual es sustento de vida de una población considerable. Por lo tanto, la estimación energética realizada ha sido solamente en base al componente orgánico, RSOD. La generación de energía usando los RSOD, al año 2017, resultó en 15,33 $M W$ aproximadamente y se estimó que aumentaría a 16,60 $M W$ en 10 años (incremento de $8 \%$ ). De ser implementada la generación de energía a partir de los RSOD a través de la incineración, esta representaría aproximadamente el 8,66 \% (al 2017) de la producción energética por biomasa en el Perú ${ }^{15}$.

El beneficio de transformar los RSOD en energía, va a permitir reemplazar porcentualmente el uso de los combustibles fósiles. Asimismo, se evitaría las enfermedades de efecto biológico por el inadecuado manejo de estos. Además, se disminuiría las emisiones de metano al medio ambiente y se aumentaría la vida útil de los botaderos municipales al disminuir la cantidad de residuos depositados.

\section{AGRADECIMIENTOS}

A la UNS, por su apoyo a través del Fondo Especial de Desarrollo Universitario (FEDU) para la ejecución de esta investigación. A los estudiantes del curso de Ingeniería Ambiental de la Escuela de Ingeniería Mecánica de la UNS, por contribuir en la recolección de los RSD, en especial a André Rodríguez Baltazar, Encarnación Ocaña Sánchez y a Luis Cabanillas Castillejo. Expresamos también nuestro agradecimiento al Organismo de Evaluación y Fiscalización Ambiental (OEFA) - Chimbote, en especial al Ing. Edwin Flores Castillo, por sus fructíferas discusiones en el análisis de los resultados. Agradecemos también al Laboratorio de Fisicoquímica de la UNS por contribuir con las mediciones del poder calorífico de los RSOD.

\section{REFERENCIAS BIBLIOGRÁFICAS}

1 Romero Salvador A. Aprovechamiento de la biomasa como fuente de energía alternativa a los combustibles fósiles. Rev. R. Acad. Cienc. Exact. Fis. Nat. 2010; 104(2): 331-345.

2 Kumar A., Samadder S.R. A review on technology options of waste to energy for effective management of municipal solid waste. Waste Management. 2017; 69: 407-422.

3 Vásquez O.C. Gestión de los residuos sólidos municipales en la ciudad del Gran Santiago de Chile: Desafíos y oportunidades. Rev. Int. Contam. Ambie. 2011; 27(4): 347-355.

4 PEAER. Plan Nacional de Desarrollo 2013-2018: El programa especial para el aprovechamiento de energías renovables. Estados Unidos Mexicanos. [Consultado el 17 de noviembre del 2017]. Disponible en: https://www.gob.mx/cms/uploads/attachment/ file/249/PEAER-2014.pdf.

5 MINAM-Sigersol. Sistema de información para la gestión de residuos sólidos: Informe anuario 2011-2015. 
6 Arvizu Fernández J.L. La basura como recurso energético: situación actual y prospectiva en México. Rev. Ing. Civil. 2011; 496: 36-44.

7 Topanou N., Domeizel M, Fatombi J., Josse R.G., Aminou T. Characterization of household solid waste in the town of Abomey-Calavi in Benin. Journal of Environmental Protection. 2011; 2(6): 692-699.

8 Miyashiro L., Mazuelos G., Toledo E., Vega R. Estudio de mercado de la vivienda social en la ciudad de Chimbote. Fondo Mi Vivienda. 2009; 74 pp.

9 MINAM. Guía metodológica para el desarrollo del Estudio de Caracterización para Residuos Sólidos Municipales (EC-RSM). [Consultado el 18 de diciembre del 2017]. Disponible en: http://redrrss.minam.gob.pe/material/20150302182233.pdf.

10 Registro Nacional de Municipalidades 2016. Informe técnico 2. [Consultado el 14 de febrero del 2018]. Disponible en: https://www.inei.gob.pe/media/MenuRecursivo/ boletines/informe-tecnico-n-2-registro-nacional-de-municipalidades-2016.pdf.

11 Oviedo J., Coral K., Cifrian E., Andrés A. Valorización energética de los RSU de la parroquia Limoncocha-reserva biológica ecuador. 2015-2016. VII Simposio de Iberoamericano en Ingeniería de Residuos. 13-14 de julio de 2017. Santander-España. 745-750.

12 Tugov A.N. Experience of Using Municipal Solid Waste in the Energy Industry (An Overview). Thermal Engineering. 2015; 62(12): 853-851.

13 Romero Salvador A. La incineradora de residuos: ¿está justificado el rechazo social? Rev. R. Acad. Cienc. Exact. Fis. Nat. 2010; 104(1): 175-187.

14 Municipalidad de Ate. Estudio de caracterización de residuos sólidos domiciliarios del distrito de Ate. ECI, IPES.2011. 84 pp.

15 MINEM. Anuario ejecutivo de electricidad 2014. Dirección de Estudios y Promoción Eléctrica. [Consultado el 23 de febrero del 2018]: http://www.minem.gob.pe/_ publicaSector.php?idSector $=6$. 